\title{
A positron emission tomography study of cerebrovascular reserve before and after shunt surgery in patients with idiopathic chronic hydrocephalus
}

\author{
Petra M. Klinge, M.D., Georg Berding, M.D., Ph.D., Thomas Brinker, M.D., Ph.D., \\ Wolfram H. KnaPp, M.D., Ph.D., ANd MadJID Samit, M.D., Ph.D. \\ Departments of Neurosurgery and Nuclear Medicine, Medical School Hannover, and Department of \\ Neurosurgery, Nordstadt Hospital Hannover, Hannover, Germany
}

\begin{abstract}
Object. In this study the authors use positron emission tomography (PET) to investigate cerebral blood flow $(\mathrm{CBF})$ and cerebrovascular reserve (CVR) in chronic hydrocephalus.

Methods. Ten patients whose mean age was $67 \pm 10$ years (mean \pm standard deviation [SD]) were compared with 10 healthy volunteers who were $25 \pm 3$ years of age. Global CBF and CVR were determined using ${ }^{15} \mathrm{O}-\mathrm{H}_{2} \mathrm{O}$ and PET prior to shunt placement and 7 days and 7 months thereafter. The CVR was measured using $1 \mathrm{~g}$ acetazolamide. Neurological status was assessed based on a score assigned according to the methods of Stein and Langfitt.

Seven months after shunt placement, five patients showed clinical improvement (Group A) and five did not (Group B). The average global CBF before shunt deployment was significantly reduced in comparison with the control group $(40 \pm 8$ compared with $61 \pm 7 \mathrm{ml} / 100 \mathrm{ml} /$ minute; mean $\pm \mathrm{SD}, \mathrm{p}<0.01)$. In Group A the CBF values were significantly lower than in Group B (36 \pm 7 compared with $44 \pm 8 \mathrm{ml} / 100 \mathrm{ml} / \mathrm{minute} ; \mathrm{p}<0.05)$. The CVR before surgery, however, was not significantly different between groups (Group A $=43 \pm 21 \%$, Group B $=37 \pm 29 \%$ ). After shunt placement, there was an increase in the CVR in Group A to $52 \pm 37 \%$ after 7 days and to $68 \pm 47 \%$ after 7 months $(\mathrm{p}<0.05)$, whereas in Group B the CVR decreased to $14 \pm 18 \%(\mathrm{p}<0.05)$ after 7 days and returned to the preoperative level $(39 \pm 6 \%) 7$ months after shunt placement.

Conclusions. The preliminary results indicate that a reduced baseline CBF before surgery does not indicate a poor prognosis. Baseline CBF before shunt placement and preoperative CVR are not predictive of clinical outcome. A decrease in the CVR early after shunt placement, however, is related to poor late clinical outcome, whereas early improvement in the CVR after shunt placement indicates a good prognosis.
\end{abstract}

\section{KEY WORDS • chronic hydrocephalus positron emission tomography}

The diagnosis of hydrocephalus is commonly based on neurological examination, computerized tomography (CT) scanning, or magnetic resonance tomography, and the assessment of cerebrospinal fluid (CSF) dynamics. Additionally, measurement of cerebral blood flow (CBF) has been believed to facilitate the surgical decisionmaking process. Cortical mapping of $\mathrm{CBF}$ was predominantly performed using single photon emission studies. ${ }^{3,11,17,21,28}$ The results were inconsistent: Larsson, et al., ${ }^{17}$ stated that the ratio between the perfusion in the frontal and that in the occipital region was helpful in prognostication. Tanaka, et al. ${ }^{43}$ postulated that impairment of global CBF in patients with hydrocephalus indicates poor clinical outcome after surgery for shunt placement. In contrast, in a previous positron emission tomography (PET) study ${ }^{13}$ it was suggested that a reduction in global CBF was associated with a good prognosis posttreatment.

The estimation of the cerebrovascular reserve (CVR) as a response of cerebral autoregulation is an important additional parameter for characterizing the vascular state and has been revealed to be useful in a number of clinical problems such as in trauma research ${ }^{22}$ and in cerebrovascular disease. ${ }^{2,38}$
Some data on the CVR capacity in patients presenting with symptoms of normal-pressure hydrocephalus have been reported. ${ }^{16,27,35,43,44}$ Some investigators have postulated a preserved CVR in chronic hydrocephalus. ${ }^{16,35}$ Based on Xe-CT studies, however, Meier, et al., ${ }^{27}$ reported that the CVR was preserved only in the early stages of the disease. Because the stimuli of CVR preservation were different, the results are not quite comparable: xenon inhalation, ${ }^{27}$ application of angiotensin-converting enzyme,,$^{35}$ CSF tapping, ${ }^{16}$ and administration of acetazolamide ${ }^{43}$ elic- $^{2}$ it different physiological reactions.

Because the results of CBF and CVR measurements in chronic hydrocephalus are still contested, we performed a PET investigation to study the patients' cerebrovascular status before and after shunt treatment. Because measuring CBF by using ${ }^{15} \mathrm{H}_{2} \mathrm{O}-\mathrm{PET}$ is a unique modality for performing quantitative studies in ischemic diseases of the brain, ${ }^{2,36}$ this method was used together with administration of acetazolamide for CVR mapping. ${ }^{1,9,19,32,39}$

In this study we deal with the specific question of whether $\mathrm{CBF}$ and/or CVR capacity prior to or within a short interval after surgery in patients with chronic hydrocephalus is related to the late clinical outcome of shunt treatment. 
TABLE 1

Neurological score, ICP, and CSF dynamics compared with clinical outcome in 10 patients with chronic hydrocephalus*

\begin{tabular}{lcc}
\hline \hline \multicolumn{1}{c}{ Factor } & $\begin{array}{c}\text { Group A } \\
\text { (5 patients) }\end{array}$ & $\begin{array}{c}\text { Group B } \\
(5 \text { patients })\end{array}$ \\
\hline neurological score (preop) & 9 & 7 \\
ICP $(\mathrm{mm} \mathrm{Hg})$ & $22.0 \pm 8.1$ & $22.8 \pm 14.3$ \\
$\mathrm{R}_{\text {out }}(\mathrm{mm} \mathrm{Hg} / \mathrm{ml} /$ minute) & $8.5 \pm 2.1$ & $6.3 \pm 2.5$ \\
PVI $(\mathrm{ml})$ & $47.0 \pm 40.3$ & $39.0 \pm 20.4$ \\
\hline
\end{tabular}

* Values are presented as the mean $\pm \mathrm{SD}$. The preop neurological score was assessed according to the method of Stein and Langfitt.

\section{Clinical Material and Methods}

\section{Protocol and Patient Population}

We studied five women and five men with a mean age of $67 \pm 10$ years (range 49-77 years) who had been scheduled for ventriculoperitoneal (VP) shunt placement on presentation with symptoms of chronic hydrocephalus (gait disturbances, mental deterioration, and urinary incontinence). All patients exhibited at least two of the typical symptoms, among which gait disturbances prevailed. The onset of clinical symptoms occurred at 6 months to 5 years before surgery. All patients underwent cerebral CT or magnetic resonance tomography to verify enlargement of the ventricular system; obliteration or dilation of the cerebral sulci at the convexity and/or periventricular low density were considered signs of disturbed CSF absorption. Before surgery, continuous intracranial pressure (ICP) monitoring was performed for at least 48 hours (for example, to detect pathological wave complexes). ${ }^{20}$ Additionally, the CSF outflow resistance $\left(\mathrm{R}_{\text {out }}\right)$ was measured by intrathecal infusions of sterile isotonic saline given at a constant rate of $90 \mathrm{ml} /$ hour. The pressure volume index (PVI) was evaluated as a measure of the intracranial compliance by administering intrathecal bolus injections. ${ }^{23}$

In all patients a medium-pressure valve was inserted (Pudenz-Schulte; Medtronic, Inc., Minneapolis, MN). The $\mathrm{CBF}$ was measured before surgery and approximately 1 week and 7 months postsurgery.

\section{Neurological Scoring}

Clinical outcome was assessed using neurological scoring for gait and mental disturbances according to the classification system of Stein and Langfitt, ${ }^{40}$ based on the degree of functional disturbance. Each symptom was scored with numbers ranging from 0 (none) to 5 (no capacity for independent function). Both numbers were then added, and if urinary incontinence was present another point was added to the sum. This sum was termed the "impairment score." Clinical status pre- and postsurgery was classified by the same surgeon. A decrease in the impairment score of three or more points postsurgery was regarded as clinical improvement. By this definition, five patients had improved (Group A) and five had not (Group B) at an interval of 7 months after the initial investigation.

\section{Mapping CBF With PET}

Measurements were performed under standardized conditions after the patients had rested for 15 minutes. Their

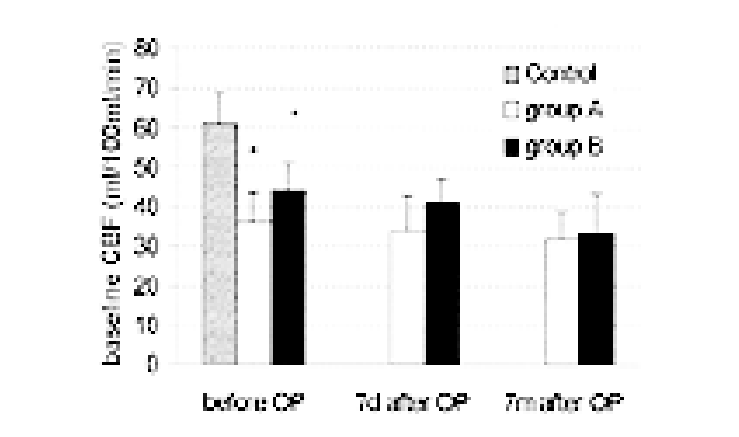

FIG. 1. Bar graph showing global baseline CBF (in $\mathrm{ml} / 100$ $\mathrm{ml} /$ minute) before shunt placement and 7 days and 7 months postsurgery in both outcome groups (Groups A and B; mean $\pm \mathrm{SD}$; $* \mathrm{p}<0.05)$. OP $=$ operation.

ears and eyes were open during the procedure. Informed consent was obtained from the patients or their guardian.

Annihilation photons were registered with a PET scanner (ECAT 951/31; Siemens, Erlangen, Germany). Thirtyone slices with a plane separation of $3.4 \mathrm{~mm}$ were obtained simultaneously. The axial and transaxial resolution of the reconstructed images (Hann-filter, cut-off frequency $0.4,128 \times 128$ matrix) was approximately 7 to $8 \mathrm{~mm}$ full width at half maximum. After a bolus injection of 3.7 $\mathrm{GBq}{ }^{15} \mathrm{O}$-labeled water, multiple sequential periods were recorded $(12 \times 5$ seconds, $4 \times 15$ seconds, $2 \times 30$ seconds, and $2 \times 60$ seconds). The total scan period was 5 minutes. The method for synthesis and the automated procedure for the application of the ${ }^{15} \mathrm{O}$-labeled water has been described previously. ${ }^{25}$ All images were corrected for attenuation (10-minute transmission) and decay during the reconstruction procedure. Radial artery blood samples (1 $\mathrm{ml}$ ) were taken in the middle of each time interval of the frame mode acquisition, except for one sample that was taken immediately before the scan. The samples were counted in a well counter that was cross-calibrated to the PET scanner. The decay-corrected values were transferred to a computer workstation (SUN 4; Sun Microsystems, Palo Alto, CA) for further data processing. The CBF was calculated (in $\mathrm{ml} / 100 \mathrm{ml} /$ minute) from the time course of tissue and blood activity by using a one-compartment Kety-Schmidt model, and displayed on parametric flow maps. Corrections for delay and dispersion of input function have been described by Van den Hoff, et al..$^{45}$ Data sets from different investigations and from different patients were reoriented using the orbitomeatal line.

Regional and global CBF analysis was based on the region-of-interest technique on a representative slice at the level of the basal ganglia. Regions of interest were manually defined as corresponding to the territories of the anterior cerebral artery, the middle cerebral artery, and the posterior cerebral artery of both hemispheres, based on the atlas of Talairach and Tournoux. ${ }^{42}$ Gray and white matter were not differentiated because the actual imaging resolution did not provide reliable identification of white matter due to the enlargement of the ventricular system.

The CVR capacity was determined after intravenous administration of $1 \mathrm{~g}$ acetazolamide over 5 minutes., ${ }^{9,19,33}$, 39,41 To guarantee CBF mapping during maximum vasodilation of cerebral vessels, repeated activity recordings were begun after an interval of 15 minutes postinjection. 
The CVR was determined as the relative difference in the $\mathrm{CBF}$ at baseline and during stimulation (percentage of change).

\section{Control Volunteers}

Reference values for global baseline CBF were obtained from 10 healthy volunteers (three women and seven men). Their ages ranged from 24 to 33 years, with a mean age of $25.7 \pm 2.9$ years. The mean $\mathrm{CBF}$ was $61 \pm$ $8 \mathrm{ml} / 100 \mathrm{ml} / \mathrm{minute}$ and ranged from 50 to $72 \mathrm{ml} / 100 \mathrm{ml} /$ minute.

\section{Statistical Analysis}

Group differences (controls and Groups A and B) and changes in baseline CBF or CVR capacity were tested by using a one- or two-tailed, paired or unpaired Student's ttest. For significant findings, the specific probability value is noted. Values are expressed as the mean $\pm 1 \mathrm{SD}$.

\section{Results}

Neurological Scoring, ICP, and CSF Dynamics Presurgery

The ICP ranged from 7 to $42 \mathrm{~mm} \mathrm{Hg}(22 \pm 11 \mathrm{~mm} \mathrm{Hg})$, the $\mathrm{R}_{\text {out }}$ from 4 to $11 \mathrm{~mm} \mathrm{Hg} / \mathrm{ml} / \mathrm{minute}(7 \pm 2 \mathrm{~mm} \mathrm{Hg} / \mathrm{ml} /$ minute), and the PVI from 14 to $93 \mathrm{ml}(43 \pm 30 \mathrm{ml})$. The neurological scores ranged from 6 to 10 (median 8).

By conventional clinical methods, that is, scoring the neurological impairment, no differences were found in the CSF dynamics $\left(\mathrm{R}_{\text {out }}\right.$ and PVI) between Groups $\mathrm{A}$ and $\mathrm{B}$ (p > 0.05; Table 1).

\section{Global CBF and CVR Presurgery}

Global CBF values in all patients were significantly reduced in comparison with the control group: $40 \pm 8$ compared with $61 \pm 8 \mathrm{ml} / 100 \mathrm{ml} /$ minute, respectively $(\mathrm{p}<$ $0.01)$. Regarding outcome groups, both Group A (36 \pm 7 $\mathrm{ml} / 100 \mathrm{ml} / \mathrm{minute})$ and Group B (44 $\pm 8 \mathrm{ml} / 100 \mathrm{ml} /$ minute) had significantly lower global CBF presurgery compared with the control group ( $\mathrm{p}>0.05$; Fig. 1). In comparing outcome groups we found that the reduced global baseline CBF in Group A was significantly different from that in Group B: $36 \pm 7$ compared with $44 \pm 8$ $\mathrm{ml} / 100 \mathrm{ml} / \mathrm{minute}$, respectively $(\mathrm{p}<0.05)$. The CVR, however, was no different in either outcome group: $43 \pm$ $21 \%$ and $37 \pm 29 \%$, respectively (Fig. 2).

\section{Global CBF and CVR Postsurgery}

Within both outcome groups global baseline CBF levels (in $\mathrm{ml} / 100 \mathrm{ml} / \mathrm{minute}$ ) did not change 7 days and 7 months postsurgery: $34 \pm 6$ and $32 \pm 10$ (Group A) and $41 \pm 8$ and $32 \pm 6$ (Group B), respectively ( $p>0.05$; Fig. 1).

In contrast with baseline CBF, CVR capacity varied. The CVR in Group A increased: $52 \pm 37 \%$ after 7 days and $68 \pm 47 \%$ after 7 months. The CVR increase after seven months was statistically significant compared with preoperative values $(43 \pm 21 \%, \mathrm{p}<0.05)$. In Group B the CVR decreased significantly within 7 days postsurgery $(14 \pm 18$ compared with $37 \pm 29 \%, \mathrm{p}>0.05)$ and re-

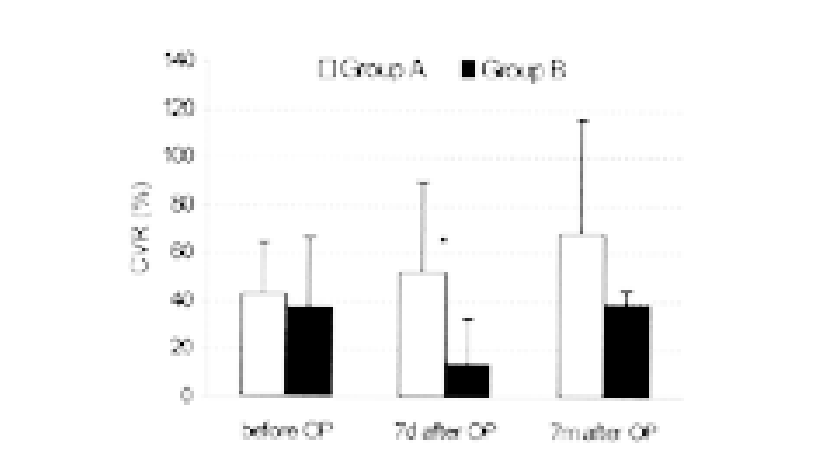

FIG. 2. Bar graph showing CVR capacity (CVR\%) after stimulus with acetazolamide before shunt placement and 7 days and 7 months postsurgery in both outcome groups (Groups A and B; mean $\left.\pm \mathrm{SD} ; *_{\mathrm{p}}<0.05\right)$. OP = operation

turned to the preoperative level after 7 months $(39 \pm 6 \%$, Fig. 2). The observed differences in CVR between outcome groups at 7 days postsurgery were significant: $14 \pm$ $18 \%$ in Group B compared with $52 \pm 37 \%$ in Group A $(\mathrm{p}<0.05)$. However, the differences in CVR after 7 months did not reach statistical significance: $39 \pm 6 \%$ in Group B and $68 \pm 47 \%$ in Group A.

The relative changes in CVR after surgery for shunt placement compared with preoperative values and expressed as the average CVR ratio were significantly different between the outcome groups: 0.45 in Group B and 1.3 in Group A $(p<0.05)$. Again, after 7 months the differences did not reach statistical significance: 1.04 in Group B and 1.72 in Group A.

\section{Discussion}

Because vascular status is regarded as a determinant for neurological status in patients with hydrocephalus, we suggest that investigators use measures of the vascular system as criteria for the selection of treatment. ${ }^{4,7}$

The CBF and CVR capacity are important parameters of the functional status of the cerebrovascular system. ${ }^{22}$ In cerebrovascular disease, local or regional deficits of CVR are characteristic alterations. ${ }^{30,36,38,41}$ Because chronic hydrocephalus may affect global CBF by impairment of CSF circulation, as indicated by experimental animal studies, ${ }^{29}$ abnormalities in $\mathrm{CBF}$ and CVR are expected to occur throughout cerebral territories. ${ }^{5,6}$ Therefore, global CBF and CVR can be assumed to reflect adequately alterations in the cerebrovascular system in chronic hydrocephalus.

In this study we questioned whether global CBF and CVR capacity can be correlated to late clinical outcome when studies are performed presurgery or within a short interval postsurgery.

We have demonstrated that global baseline CBF was significantly reduced in all patients with hydrocephalus compared with a healthy control group. Because the mean ages were different in each group, the potential influence of age on CBF must be taken into account. ${ }^{10,18,24,38}$ Leenders, et al. ${ }^{18}$ have investigated a normal population of sufficient size to correlate $\mathrm{CBF}$ with age. Using the same PET methodology, their values were in the same range as in our study.

On average, $\mathrm{CBF}$ decreased by $0.5 \%$ per year of age. 
Even if this relationship is taken into account, baseline $\mathrm{CBF}$ in patients with hydrocephalus was definitely lower (approximately $20 \%$ on average) than would be expected in an age-adjusted population.

The relevant result, however, is that low CBF values before shunt surgery did not indicate a poor prognosis, as we already suggested in our previous study. ${ }^{13}$ Instead, baseline CBF presurgery was lower in patients with good clinical outcomes than in patients who did not improve after shunt surgery. This difference cannot be explained by an age effect, because there was no significant difference in the mean ages between both outcome groups (66 compared with 69 years of age).

Furthermore, the CVR capacity before shunt deployment had no predictive value, whereas an early increase of CVR after shunt placement was related to a good prognosis. An early decrease of CVR, however, indicated poor late clinical outcome. These PET findings are all the more important because they were the only predictors of outcome: values for $\mathrm{R}_{\text {out }}$ and the PVI were not related to clinical outcome.

\section{Neurological Scoring and CBF Mapping With PET Studies}

The late clinical outcome was evaluated 7 months postsurgery because it has been shown that within the first 6 months after shunt insertion considerable changes in clinical status may occur. ${ }^{40,46}$ To classify patients in a group of responders and nonresponders we assigned a score obtained using the methods of Stein and Langfitt ${ }^{40}$ in patients with normal-pressure hydrocephalus. This score is a modified Barthels and Katz Index of activities of daily living. ${ }^{12,37}$ Because this index is subject to a certain degree of observer bias, we postulated that a minimum decrease of 3 points is required to indicate an improvement in an individual patient's neurological status. We further postulated that the neurological status had not improved when the score was not changed by more than one point or when it increased.

Follow up of global and regional CBF is not only hampered by technical limitations inherent in the different modalities used but is also influenced by varying physiological and psychological conditions and/or environmental factors present during measurement. In particular, varying activation of cortical areas as a result of the individual's mental and cognitive status may influence local distribution. ${ }^{34}$ Therefore, all possible steps were taken to standardize the conditions under which PET studies were performed (that is, a resting period before pharmaceutical injection, and so on).

Despite this standardized investigation protocol it must be assumed that subsequent investigations may result in $\mathrm{CBF}$ values showing small systematic differences. ${ }^{8,26}$ Consequently, differences observed in follow-up studies of CBF and CVR can only be assigned to therapeutic effects if there are significant differences between patients with evidence of clinical improvement and those with no improvement.

Despite the abovementioned methodological limitations the data of our preliminary study indicate that responders and nonresponders after VP shunt insertion differ in their vascular status (baseline CBF and CVR) before and after shunt treatment and furthermore that abnormal baseline CBF before surgery in patients might be associated with a favorable outcome.

In contrast to the work of Meier, et al. ${ }^{27}$ who postulated preoperative differences in CVR in patients with and without beneficial effects after VP shunt placement, we did not observe significant differences in the outcome groups regarding CVR before surgery. However, the determination of outcome by using the pretreatment CVR parameter has not been validated. ${ }^{27}$

Other contradictory findings were reported by Tanaka, et al., ${ }^{43}$ who postulated an association between clinical improvement after VP shunt insertion and preserved baseline CBF together with impaired CVR, whereas our data showed that decreased baseline CBF together with a preserved CVR is not associated with poor outcome after shunt placement. The data in their study were based on the Xe-washout method, which may be affected by the pharmacological effect of xenon and which is dependent on the individual Xe distribution volume. ${ }^{15,30}$ A vasodilatory and narcotic effect of the contrast medium has been reported. ${ }^{14,31}$

\section{Conclusions}

From our study we conclude that baseline CBF and CVR values presurgery are not definitely predictive of the clinical outcome. Early after VP shunt insertion, however, both parameters of cerebrovascular function may be useful in predicting clinical long-term response to shunt surgery. Because of the preliminary character of this study and the small number of patients included, the potential clinical utility of consecutive CBF and CVR measurement cannot yet be determined. However, it appears justified to expand the data base to investigate the predictive accuracy of CVR capacity after VP shunt placement.

\section{References}

1. Bonte F, Devous M, Reisch JS: The effect of acetazolamide on regional cerebral blood flow in normal human subjects as measured by single-photon emission computed tomography. Invest Radiol 23:564-568, 1988

2. Broich K, Alavi A, Kushner M: Positron emission tomography in cerebrovascular disorders. Semin Nucl Med 22:224-232, 1992

3. Caner H, Caner B, Turul H, et al: Pre and postoperative assessment of regional cerebral blood flow in hydrocephalus by $99 \mathrm{~m}-$ Tc-hexamethyl-propylenamine oxime SPECT. J Nucl Biol Med 35:66-72, 1991

4. Del Bigio MR: Neuropathological changes caused by hydrocephalus. Acta Neuropathol 85:573-585, 1993

5. Del Bigio MR, Bruni JE: Changes in periventricular vasculature of the rabbit brain following induction of hydrocephalus before and after shunting. J Neurosurg 69:115-120, 1988

6. Del Bigio MR, Bruni JE: Periventricular pathology in hydrocephalic rabbits before and after shunting. Acta Neuropathol 77:186-195, 1988

7. Earnest MP, Fahn S, Karp JH, et al: Normal pressure hydrocephalus and hypertensive cerebrovascular disease. Arch Neurol 31:262-266, 1974

8. Frackowiak RS, Lenzi GL, Jones T, et al: Quantitative measurement of regional cerebral blood flow and oxygen metabolism in man using 150 and positron emission tomography: theory, procedure and normal values. J Comput Assist Tomogr 4: 727-736, 1980 
9. Gambhir S, Inao S, Tadokoro M, et al: Comparison of vasodilatory effect of carbon dioxide inhalation and intravenous acetazolamide on brain vasculature using positron emission tomography. Neurol Res 19:139-144, 1997

10. Globus M, Melamed E: Progressive age-related decrease in regional cerebral blood flow in healthy subjects. Isr J Med Sci 21:662-665, 1985

11. Graff-Radford NR, Rezai K, Godersky JC, et al: Regional cerebral blood flow in normal pressure hydrocephalus. J Neurol Neurosurg Psychiatry 50:1589-1596, 1987

12. Katz S, Ford AB, Moskowitz RW, et al: Studies of illness in the aged. The index of ADL: a standardized measure of biological and psychosocial function. JAMA 185:914-919, 1963

13. Klinge P, Fischer J, Brinker T, et al: PET and CBF studies in chronic hydrocephalus: a contribution to surgical indication and prognosis. J Neuroimaging 8:205-209, 1998

14. Koeppe RA, Hutchins GD, Rothley JM, et al: Examination of assumptions for local cerebral blood flow studies in PET. J Nucl Med 28:1695-1703, 1987

15. Kreisig T, Schmiedek P, Leinsinger G, et al: 133Xe-DSPECT: Normalwerte von zerebraler Ruhedurchblutung und Reservekapazität. Nuklearmedizin 26:192-197, 1987

16. Kristensen B, Malm J, Fagerlund M, et al: Regional cerebral blood flow, white matter abnormalities, and cerebrospinal fluid hydrodynamics in patients with idiopathic adult hydrocephalus syndrome. J Neurol Neurosurg Psychiatry 60:282-288, 1996

17. Larsson A, Bergh AC, Bilting M, et al: Regional cerebral blood flow in normal pressure hydrocephalus: diagnostic and prognostic aspects. Eur J Nucl Med 21:118-123, 1994

18. Leenders KL, Perani D, Lammertsma AA, et al: Cerebral blood flow, blood volume and oxygen utilization. Normal values and effect of age. Brain 113:27-47, 1990

19. Leinsinger G, Piepgras A, Einhäupl K, et al: Normal values of cerebrovascular reserve capacity after stimulation with acetazolamide measured by xenon 133 single-photon emission CT. AJNR 15:1327-1332, 1994

20. Lundberg N: Continuous recording and control of ventricular fluid pulse pressure in neurosurgical practice. Acta Psychiatr Neurol Scand 36 (Suppl 149): 1-193, 1960

21. Mamo HL, Meric PC, Ponsin JC, et al: Cerebral blood flow in normal pressure hydrocephalus. Stroke 18:1074-1080, 1987

22. Marion DW, Darby J, Yonas H: Acute regional cerebral blood flow changes caused by severe head injuries. J Neurosurg 74: 407-414, 1991

23. Marmarou A, Shulman K, Rosende RM: A nonlinear analysis of the cerebrospinal fluid system and intracranial pressure dynamics. J Neurosurg 48:332-344, 1978

24. Martin AJ, Friston KJ, Colebatch JG, et al: Decreases in regional cerebral blood flow with normal aging. J Cereb Blood Flow Metab 11:684-689, 1991

25. Matzke KH, Meyer GJ, Hundeshagen H: An advanced system for the administration of ${ }^{15} \mathrm{O}$-water. J Labelled Comp Radiopharm 32:459-460, 1993 (Abstract)

26. Mazziotta JC, Phelps ME: Positron emission tomography studies of the brain, in Phelps ME, Mazziotta JC, Schelbert H (eds): Positron Emission Tomography and Autoradiography: Principles and Applications for the Brain and Heart. New York: Raven Press, 1986, pp 493-579

27. Meier U, Reichmuth B, Zeilinger FS, et al: The importance of xenon-computed-tomography in the diagnosis of normal pressure hydrocephalus. Int J Neuroradiol 2:153-160, 1996

28. Moretti JL, Sergent A, Louarn F, et al: Cortical perfusion assessment with ${ }^{123}$ I-isopropyl amphetamine ( ${ }^{123}$ I-IAMP) in nomal pressure hydrocephalus (NPH). Eur J Nucl Med 14:73-79, 1988

29. Nakada J, Oka N, Nagahori T, et al: Changes in the cerebral vascular bed in experimental hydrocephalus: an angio-architectural and histological study. Acta Neurochir 114:43-50, 1992
30. Nighogossian N, Trouillas P, Philippon B, et al: Cerebral blood flow reserve assessment in symptomatic versus asymptomatic high-grade internal carotid artery stenosis. Stroke 25: 1010-1013, 1994

31. Obrist WD, Jaggi JL, Smith DS, et al: Effect of stable xenon inhalation on human CBF. J Cereb Blood Flow Metab 5 (Suppl 1):S557-S558, 1985

32. Pinard E, Mazoyer B, Verrey B, et al: Rapid measurement of regional cerebral blood flow in the baboon using 150-labelled water and dynamic positron emission tomography. Med Biol Eng Comput 31:495-502, 1993

33. Posner JB, Plum F: The toxic effects of carbon dioxide and acetazolamide in hepatic encephalopathy. J Clin Invest 39: 1246-1258, 1960

34. Roland PE: Brain Activation. New York: John Wiley \& Sons, 1993, pp 469-486

35. Schmidt JF, Andersen AR, Paulson OB, et al: Angiotensin converting enzyme inhibition, $\mathrm{CBF}$ autoregulation, and ICP in patients with normal-pressure hydrocephalus. Acta Neurochir 106:9-12, 1990

36. Sette G, Baron JC, Mazoyer B, et al: Local brain haemodynamics and oxygen metabolism in cerebrovascular disease. Brain 112:931-951, 1989

37. Shah S, Vanclay F, Cooper B: Improving the sensitivity of the Barthel Index for stroke rehabilitation. J Clin Epidemiol 42: 703-709, 1989

38. Shaw TG, Mortel KF, Meyer JS, et al: Cerebral blood flow changes in benign aging and cerebrovascular disease. Neurology 34:855-862, 1994

39. Sorteberg W, Lindegaard KF, Rootwelt K, et al: Effect of acetazolamide on cerebral artery blood velocity and regional cerebral blood flow in normal subjects. Acta Neurochir 97: 139-145, 1989

40. Stein SC, Langfitt TV: Normal-pressure hydrocephalus. Predicting the results of cerebrospinal fluid shunting. J Neurosurg 41:463-470, 1974

41. Sullivan HG, Kingsbury TB IV, Morgan ME, et al: The rCBF response to Diamox in normal subjects and cerebrovascular disease patients. J Neurosurg 67:525-534, 1987

42. Talairach J, Tournoux P: Co-Planar Stereotaxis Atlas of the Human Brain: 3-Dimensional Proportional System. An Approach to Cerebral Imaging. New York: Thieme, 1988

43. Tanaka A, Kimura M, Nakayama Y, et al: Cerebral blood flow and autoregulation in normal pressure hydrocephalus. Neurosurgery 40:1161-1167, 1997

44. Tanaka K, Yonekawa Y, Miyake H, et al: [Idiopathic normal pressure hydrocephalus in elderly patients: its pathophysiology and diagnosis.] No Shinkei Geka 21:403-408, 1993 (Jpn)

45. Van den Hoff J, Burchert W, Müller-Schauenburg W, et al: Accurate local blood flow measurements with dynamic PET: fast determination of input function delay and dispersion by multilinear minimization. J Nucl Med 34:1770-1777, 1993

46. Vanneste JAL: Three decades of normal pressure hydrocephalus: are we wiser now? J Neurol Neurosurg Psychiatry 57: 1021-1025, 1994

Manuscript received January 3, 2000.

Accepted in final form January 14, 2000.

Address reprint requests to: Petra M. Klinge, M.D., Department of Neurosurgery, 7240, Medical School Hannover, Carl-NeubergStrasse 1, 30625 Hannover, Germany.

This manuscript was previously published in the Journal of Neurosurgery, Volume 91, pp 605-609, 1999. 